EDITORIAL

\title{
Assessing prosthetic mitral valve regurgitation by transoesophageal echo/Doppler
}

\section{P S Rahko}

\section{Transoesophageal echocardiography has greatly improved our ability to detect structural and regurgitant abnormalities associated with prosthetic mitral valves}

$\mathrm{D}$ espite advances in imaging, the assessment of a mechanical prosthetic mitral valve remains a considerable challenge, particularly when valvar regurgitation is suspected. Transthoracic imaging is well suited to assess the haemodynamics of forward flow across prosthetic mitral valves. Thus detection of prosthetic stenosis is usually accomplished without difficulty. In contrast, because of the location of the mitral valve prosthesis, the reflective characteristics of the materials used to manufacture the valve, the distance from the transducer, and the shadowing produced by the valve apparatus, detection of structural and regurgitant abnormalities of the valve is limited. Transoesophageal echocardiography (TOE) has greatly improved our ability to directly detect structural and regurgitant abnormalities associated with a prosthetic mitral valve. Studies directly comparing the efficacy of transthoracic echocardiography and TOE have established TOE as the "gold standard" for non-invasive diagnosis of prosthetic mitral valve dysfunction. ${ }^{12}$

\section{MITRAL PROSTHETIC FORWARD FLOW}

Recognising the limitations of direct transthoracic imaging of prosthetic regurgitation, recent studies have focused on characteristics of mitral prosthetic forward flow as a clue to the presence of significant regurgitation. The most successful parameters take into account the fact that high volumes of flow should cross the mitral valve in a forward direction whenever significant prosthetic regurgitation is present. An analysis of 57 patients by Olmos and colleagues ${ }^{3}$ evaluated several variables of forward flow across a prosthetic mitral valve. The most useful parameters for detecting moderate or severe mitral insufficiency were: a peak mitral velocity greater than $1.9 \mathrm{~m} / \mathrm{s}$ ( sensitivity $90 \%$, specificity $89 \%$ ); a mean gradient across the prosthetic valve greater than $5 \mathrm{~mm} \mathrm{Hg}$ (sensitivity $90 \%$, specificity $70 \%$ ); and a ratio $>2.5$ of the time velocity integral of mitral flow divided by the time velocity integral of left ventricular outflow tract flow (sensitivity $89 \%$, specificity $91 \%$ ). When combined with a pressure half time appropriate to a non-stenotic prosthesis these parameters effectively screened out patients with only minimal amounts of mitral insufficiency. While these indices help
Heart 2004;90:476-478. doi: 10.1136/hrt.2003.026484

overcome issues of shadowing they lack the ability to directly interrogate regurgitant flow, thus TOE is still necessary to define the type, location, severity, and anatomic correlates of prosthetic valve regurgitation.

TOE is exquisitely sensitive for the detection of valvar insufficiency. Indeed many cases of mild insufficiency detected by TOE are not detectable by angiography. ${ }^{2}$ Furthermore, all commonly manufactured low profile prosthetic valves have a built-in amount of "normal" regurgitant flow to help reduce the likelihood of valve thrombosis. Because of differences in design, each type of prosthetic valve has a characteristic signature of regurgitant flow that should be considered normal. ${ }^{4}$ These normal flow patterns comprise two components, the initial being the closure backflow occurring as the leaflet rapidly closes, and the more long lasting being holosystolic backflow leakage. Careful orientation of the TOE transducer to the valve and use of multiple imaging planes are important to fully differentiate pathologic regurgitation from the physiologic signature regurgitation. In general, pathologic regurgitant jets are those that are greater than $2 \mathrm{~cm}$ in length, holosystolic with turbulence, and of different shape from the expected signature physiologic regurgitant pattern of the valve. ${ }^{14}$

Following identification of a pathologic regurgitant jet, it is important to differentiate the origin of the jet as being valvar or paravalvar. This is best done by careful multiplane imaging of the valve apparatus. Tracing the origin of the regurgitant jet as being inside or outside the sewing ring is the critical step. Indeed, the most common error made in the diagnosis of mitral prosthetic regurgitation is the failure to differentiate a paravalvar leak from a valvar leak. A recent study, comprising predominantly of TOE cases, identified an error rate for this of approximately $10 \% .^{5}$ Virtually no other types of diagnostic errors were identified in this study. The authors concluded that the failure to differentiate paravalvar from valvar insufficiency was predominately due to inadequate orientation of the angle of interrogation with the prosthetic valve plane. It appeared most important to obtain a perpendicular orientation through the centre of the valve orifice to best differentiate the pathologic from physiologic regurgitation.

\section{SEVERITY OF MITRAL REGURGITATION}

Rating the severity of prosthetic mitral regurgitation is also a considerable challenge. The volume of the regurgitant flow jet is determined by the size of the regurgitant orifice, the driving force from the pressure gradient across the 
orifice, and the length of time of systole. Initial studies that defined regurgitant severity focused on characterisation of the colour flow jet. Methods that measured jet area or jet length, while initially promising, were soon found to have significant limitations because of variations in haemodynamics, instrument settings, receiving chamber characteristics, jet eccentricity, jet impingement on chamber walls, and variability in perception by readers. This led to investigation of other flow phenomena of regurgitation, particularly that related to flow convergence of the regurgitant jet into the regurgitant orifice and characterisation of the jet as it immediately exited the orifice (assessment of the vena contracta). ${ }^{67}$ Refinement of these techniques led to quantitative parameters relating the width of the vena contracta to severity, and the calculation of an effective regurgitant orifice and effective regurgitant volume. ${ }^{6-9}$ An alternative and indirect approach assessed flow phenomena in the pulmonary veins and systole reversal of flow was recognised as indicating severe mitral insufficiency. ${ }^{10}$

Despite such a wide array of parameters being available, little organised information exists on their efficacy in patients with mechanical prosthetic valves. Thus the study of Vitarelli and colleagues in this edition of Heart is a welcome addition to the literature. ${ }^{11}$ These authors studied 47 patients with clinically suspected prosthetic valve dysfunction, all of whom had various types of mechanical prosthetic valves. TOE examinations were performed across the entire $180^{\circ}$ spectrum of a multiplane transducer. An important aspect of this study was the authors' simultaneous evaluation of several parameters of regurgitant severity: mitral regurgitant area, diameter of the vena contracta, assessment of flow convergence parameters including maximal instantaneous regurgitant flow and regurgitant orifice area, and the ratio of peak systolic to peak diastolic pulmonary venous flow velocity. The efficacy of these parameters was compared against the standard angiographic $1+$ to $4+$ grading system of mitral regurgitation.

The authors found that their echocardiographic parameters worked quite well compared to angiography. The vena contracta measurement showed the highest correlation and also had the best receiver operating curve for detecting severe valvar insufficiency. Indices derived from flow convergence also performed very well. Consistent with previous studies, measurements of pulmonary venous flow characteristics and regurgitant jet area were less effective. The authors also importantly confirmed prior data showing that the use of multiple imaging planes improved the reliability of their results. ${ }^{12}$

What should be the approach to prosthetic mitral valve insufficiency in 2004? When prosthetic dysfunction is suspected it still may be reasonable in many circumstances to start with a transthoracic study. A typical comprehensive transthoracic examination in some circumstances may identify significant regurgitation. The primary danger is underestimation of regurgitant severity. To reduce dependence on direct imaging of the regurgitant jet, it is worthwhile to calculate the transthoracic forward flow parameters proposed by Olmos and colleagues. ${ }^{3}$ An assessment of peak mitral valve velocity, mean gradient, and the flow velocity integral ratio of mitral flow to left ventricular outflow tract flow is a very logical first step in screening for valvar insufficiency and utilises easily obtained data from the routine exam. If the clinical index of suspicion is low, the prosthetic valve flow characteristics are all within normal ranges, and valve morphology appears normal, evaluation may be stopped at this point. If, on the other hand, any of the other Olmos criteria are abnormal or clinical suspicion is still significant the patient should have a TOE. Those patients with a high clinical suspicion for prosthetic valvar insufficiency should go directly to TOE.

\section{TOE EXAMINATION}

The TOE examination needs to be complete and deliberate. Careful alignment of the transducer is essential to fully display leaflet motion as comprehensively as possible. Multiplane imaging should be done at a minimum of every $30^{\circ}$ from $0-180^{\circ}$. If pathologic flow is observed over and above the usual regurgitant signature of the valve it should be carefully characterised in multiple planes. Vitarelli and colleagues show that the best correlation occurs when the maximal regurgitant jet characteristics are calculated after evaluating multiple views. ${ }^{11}$ Localisation of the jet is also important for the surgeon. The decision to operate and the effectiveness of the operation can be enhanced by careful communication of the precise location of the regurgitant jet and whether or not it is paravalvar or valvar. To this end, the quantification system established by Foster and colleagues for describing the location of regurgitant leaks on both native and prosthetic valves is of considerable value. ${ }^{13}$ This system uses not only a full rotational examination but also advocates further imaging at $0^{\circ}$ sweeping through the valve by moving the transducer upward and downward in the oesophagus to show as much of the circumference of the valve plane as possible.

For characterising regurgitant severity, use of all of the major parameters investigated by Vitarelli and colleagues is recommended. ${ }^{11}$ Measurement of the width of the vena contracta, interrogation of the regurgitant jet with continuous wave Doppler, and evaluation of flow convergence parameters and perhaps even valve regurgitant jet area can be done quickly during the examination and calculated easily at the conclusion of the study. The most convincing information about regurgitant severity will occur when the conclusions reached by measuring these multiple parameters agree with each other.

What of the future? Three and four dimensional imaging reconstruction is feasible for mitral regurgitant jets. Three dimensional displays may add further important information for the surgeon in planning the operation and localising the source of the problem. ${ }^{14}$ Perhaps three dimensional display may also add quantitative information. As more rapid real time three dimensional systems become available this form of imaging will become a practical reality. For the time being, however, comprehensive two dimensional imaging combined with multiple measurements of regurgitant severity is the standard of care.

\section{REFERENCES}

1 Daniel LB, Grigg LE, Weisel RD, et al. Comparison of transthoracic and transesophageal assessment of prosthetic valve dysfunction. Echocardiography 1990;7:83-95.

2 Bach DS. Transesophageal echocardiographic (TEE) evaluation of prosthetic valves. Cardiol Clin 2000;18:751-71.

3 Olmos L, Salazar G, Barbetseas J, et al. Usefulness of transthoracic echocardiography in detecting significant prosthetic mitral valve regurgitation. Am J Cardiol 1999;83:199-205.

4 Flachskampf FA, O'Shea JP, Griffin BP, et al. Patterns of normal transvalvular regurgitation in mechanical valve prostheses. J Am Coll Cardiol 1991; 18:1493-98.

5 Faletra F, Constantin C, De Chiara F, et al. Incorrect echocardiographic diagnosis in patients with mechanical prosthetic valve dysfunction: correlation with surgical findings. Am J Med 2000;108:531-7.

6 Grayburn PA, Fehske W, Omran H, et al. Multiplane transesophageal echocardiographic assessment of mitral regurgitation by Doppler color flow mapping of the vena contracta. Am J Cardiol 1994;74:912-7.

7 Cohen GI, Davison MB, Klein AL, et al. A comparison of flow convergence with other transthoracic echocardiographic indexes of prosthetic mitral regurgitation. J Am Soc Echocardiogr 1992;5:620-7.

8 Tribouilloy C, Shen WF, Quere JP, et al. Assessment of severity of mitral regurgitation by measuring regurgitant jet width at its origin with transesophageal Doppler color flow imaging. Circulation 1992;85:1248-53.

9 Thomas JD. How leaky is that mitral valve? Simplified Doppler methods to measure regurgitant orifice area. Circulation 1997;95:548-50.

10 Klein AL, Obarski TP, Stewart WJ, et al. Transesophageal Doppler echocardiography of pulmonary venous flow: a new marker of mitral regurgitation severity. J Am Coll Cardiol 1991;18:518-26. 
11 Vitarelli A Conde $Y$, Cimino $E$, et al. Assessment of severity of mechanical prosthetic mitral regurgitation by transoesophageal echocardiography. Heart 2004;90:539-44.

12 Flachskampf FA, Hoffmann R, Franke A, et al. Does multiplane transesophageal echocardiography improve the assessment of prosthetic valve regurgitation? J Am Soc Echocardiogr 1995;8:70-8.
13 Foster GP, Isselbacher EM, Rose GA et al Accurate localization of mitral regurgitant defects using multiplane transesophageal echocardiography. Ann Thorac Surg 1998;65:1025-31.

14 De Simone R, Glombitza G, Vahl CF, et al. Assessment of mitral regurgitant jets by three-dimensional color Doppler. Ann Thorac Surg 1999:67:494-9.

\section{IMAGES IN CARDIOLOGY}

doi: $10.1136 /$ hrt.2003.023523

\section{Congenital coronary artery anomaly demonstrated by three dimensional 16 slice spiral CT angiography}

A

57 year old man with multiple risk factors for coronary artery disease presented with exercise related chest pain. An $x$ ray angiogram revealed no coronary atherosclerosis but, rather, an anomalous left coronary artery course. Whether the anomalous coronary artery travelled a clinically benign course posterior to the aorta or a potentially lethal course between the aorta and pulmonary trunk, however, could not be ascertained by the two dimensional $x$ ray angiography. Because of severe bradycardia (heart rate 47 beats per minute), causing prolonged breath-hold times, a magnetic resonance coronary angiogram was limited by extensive respiratory motion artefact and failed to clarify the route of the anomalous coronary artery. Consequently, the patient underwent coronary computed tomographic (CT) angiography. On a Siemens 16 slice CT scanner, the entire heart was scanned by using $12 \times 0.75 \mathrm{~mm}$ collimation spiral scanning and retrospective ECG-gating technique.

The left main coronary artery had an anomalous origin from the right, anterior sinus of Valsalva originating just above the right coronary artery ostium and followed a benign, retroaortic course (black arrowhead) after which it bifurcated into the left circumflex artery and a small left anterior descending artery (lower panels A-C: Ao, aorta; GCV, great cardiac vein; LA, left atrium; LAA, left atrial appendage; LAD, left anterior descending artery; LCX, left circumflex artery; LM, left main coronary artery; LV, left ventricle; PDA, posterior descending artery; PT, pulmonary trunk; RA, right atrium; RCA, right coronary artery; $\mathrm{RV}$, right ventricle; RVB, right ventricular branch). The first septal perforating artery (white arrowhead) arose just anterior to the right coronary artery ostium (upper panels $\mathrm{A}$ and $\mathrm{B}$ ), consistent with the $x$ ray angiogram. The dominant right coronary artery bifurcated into the posterior descending artery and right ventricular branch at its mid distal portion. As CT findings excluded malignant coronary anomaly, there was no need for surgical intervention.
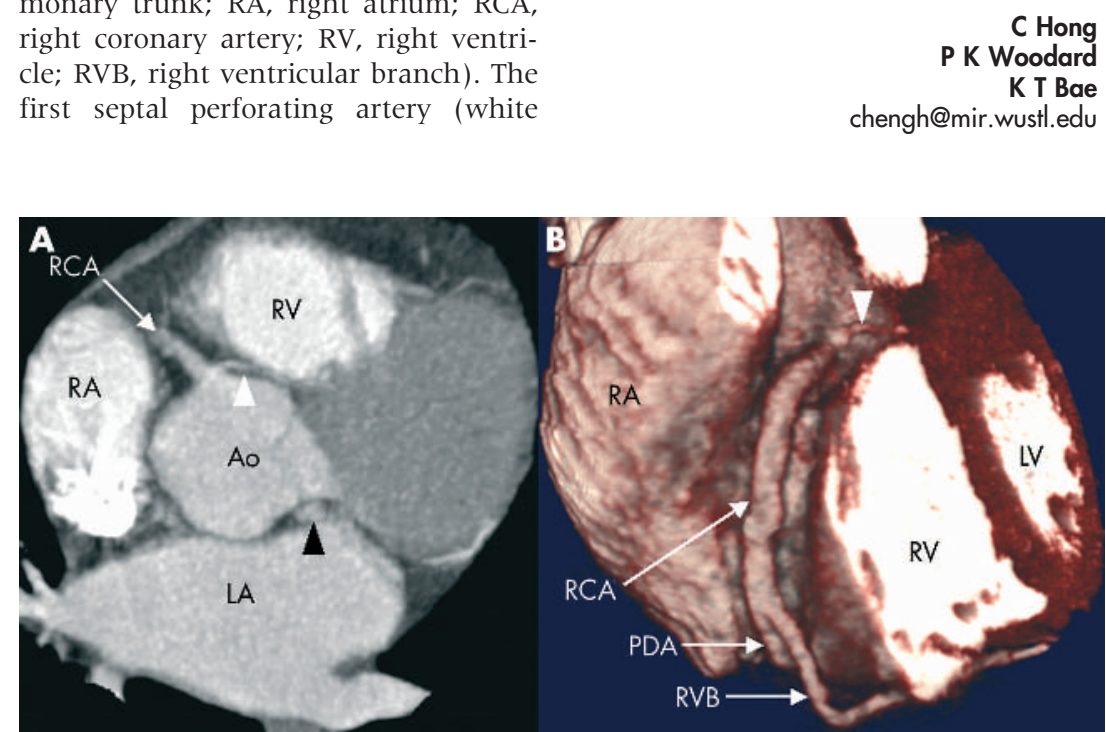

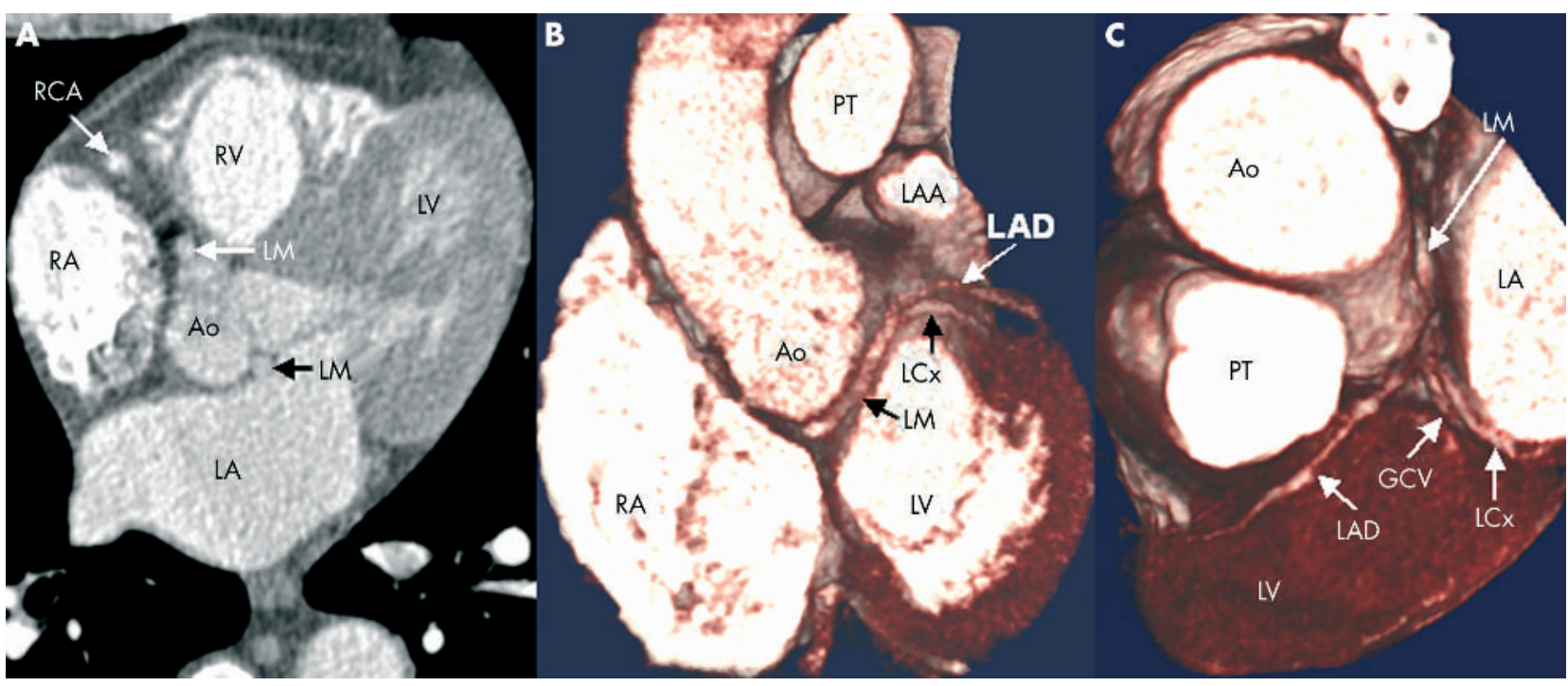

